\title{
The Architecture of Human Memory: Insights from Human Single-Neuron Recordings
}

\author{
(ㄴ)li Rutishauser, ${ }^{1,2,3}{ }^{\circledR}$ Leila Reddy, ${ }^{4,5}{ }^{\circledR}$ Florian Mormann, ${ }^{6}$ and ${ }^{\circledR}$ Johannes Sarnthein ${ }^{7,8}$ \\ ${ }^{1}$ Department of Neurosurgery, Cedars-Sinai Medical Center, Los Angeles, California 90048, ${ }^{2}$ Center for Neural Science and Medicine, Department \\ of Biomedical Sciences, Cedars-Sinai Medical Center, Los Angeles, California 90048, ${ }^{3}$ Division of Biology and Biological Engineering, California \\ Institute of Technology, Pasadena, California 91125, ${ }^{4}$ Université de Toulouse, Centre de Recherche Cerveau et Cognition, Toulouse, 31052 , France, \\ ${ }^{5}$ Centre National de la Recherche Scientifique, Toulouse, 31052, France, ${ }^{6}$ Department of Epileptology, University Hospital Bonn, 53105 Bonn, \\ Germany, ${ }^{7}$ Klinik für Neurochirurgie, Universitäts Spital und Universität Zürich, 8091 Zürich, Switzerland, and ${ }^{8}$ Neuroscience Center Zurich, \\ Universität Zürich und ETH Zürich, 8057 Zürich, Switzerland
}

Deciphering the mechanisms of human memory is a central goal of neuroscience, both from the point of view of the fundamental biology of memory and for its translational relevance. Here, we review some contributions that recordings from neurons in humans implanted with electrodes for clinical purposes have made toward this goal. Recordings from the medial temporal lobe, including the hippocampus, reveal the existence of two classes of cells: those encoding highly selective and invariant representations of abstract concepts, and memory-selective cells whose activity is related to familiarity and episodic retrieval. Insights derived from observing these cells in behaving humans include that semantic representations are activated before episodic representations, that memory content and memory strength are segregated, and that the activity of both types of cells is related to subjective awareness as expected from a substrate for declarative memory. Visually selective cells can remain persistently active for several seconds, thereby revealing a cellular substrate for working memory in humans. An overarching insight is that the neural code of human memory is interpretable at the single-neuron level. Jointly, intracranial recording studies are starting to reveal aspects of the building blocks of human memory at the single-cell level. This work establishes a bridge to cellular-level work in animals on the one hand, and the extensive literature on noninvasive imaging in humans on the other hand. More broadly, this work is a step toward a detailed mechanistic understanding of human memory that is needed to develop therapies for human memory disorders.

Key words: single-neuron; human memory; hippocampus; amygdala; entorhinal cortex; episodic memory

\section{Introduction}

How is it that reminiscing about a salient autobiographical event results in the phenomenological experience of "remembering" (Tulving, 2002) that is accompanied by a multitude of associated memories of what we were feeling at the time, what we were smelling, and what someone said to us? Unique insight into these fundamental questions has been gained by recording from single neurons in human patients implanted with electrodes for clinical purposes (Engel et al., 2005; Suthana and Fried, 2012; Johnson and Knight, 2015; Rutishauser, 2019). This technique allows investigators to observe the activity of individual neurons in areas of the human brain critical to memory (Fried et al., 2014) while patients perform tasks in which they report their subjective

\footnotetext{
Received June 30, 2020; revised Sep. 24, 2020; accepted Sep. 27, 2020.

U.R. was supported by National Institutes of Health R01 MH110831, U01NS117839, and P50MH094258, National Science Foundation Career Award BCS-1554105, and McKnight Foundation for Neuroscience. J.S. was supported by Swiss National Science Foundation SNSF 320030_176222. F.M. was supported by Volkswagen Foundation, German Ministry of Education and Research BMBF 031L0197B, and German Research Council DFG M0 930/8-1, M0 930/4-2, SFB 1089. LR was supported by French ANR-18-CE37-0007-01 Al-REPS. We thank Adam Mamelak, Jan Kamiński, and Ralph Adolphs for discussion.

The authors declare no competing financial interests.

Correspondence should be addressed to Ueli Rutishauser at ueli.rutishauser@cshs.org.

https://doi.org/10.1523/JNEUROSCI.1648-20.2020

Copyright $(2021$ the authors
}

experience, such as with what confidence they believe a face shown is familiar. This work is starting to reveal the building blocks for human memory (Quiroga, 2012, 2019; Rutishauser, 2019) and thereby identifies features of memory organization that may be uniquely human and others that are shared with other species, thereby building a bridge to the broader literature.

In this review, we highlight advances in our understanding of human memory derived from invasive single-neuron recordings in the human hippocampus, amygdala, parahippocampal gyrus, and entorhinal cortex. These parts of the medial temporal lobe (MTL) (Squire et al., 2004; Eichenbaum et al., 2007) are critical for forming and retrieving episodic memories of the "where, what, when" of past experience. The MTL is also critical for forming and using semantic memories of facts about the world (Manns et al., 2003), which constitute the features based on which episodic memories are constructed. Using this approach, different types of neurons have been identified that, for example, signal aspects of memory content and strength. The trial-by-trial variability in the responses of these neurons explains behavior, such as whether a memory was retrieved with high or low confidence (Rutishauser et al., 2015) or whether a stimulus was consciously perceived or not (Reber et al., 2017). An overarching insight derived from the work reviewed is that features of both semantic and episodic memories are encoded at the level of 


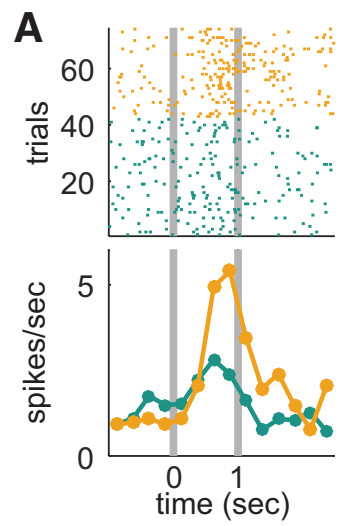

C

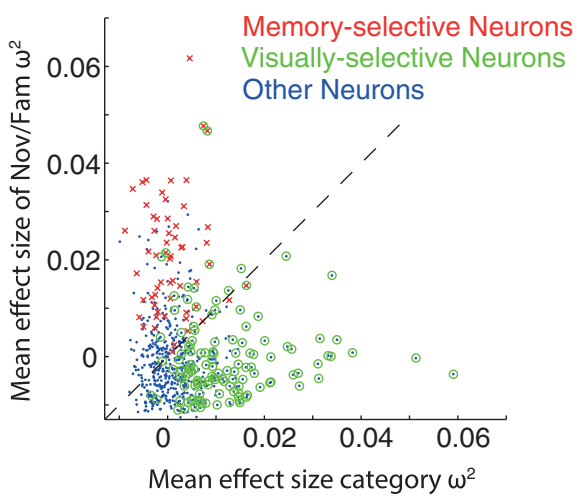

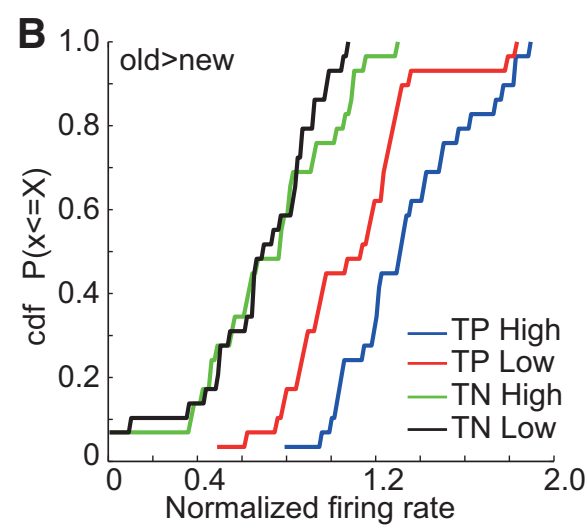

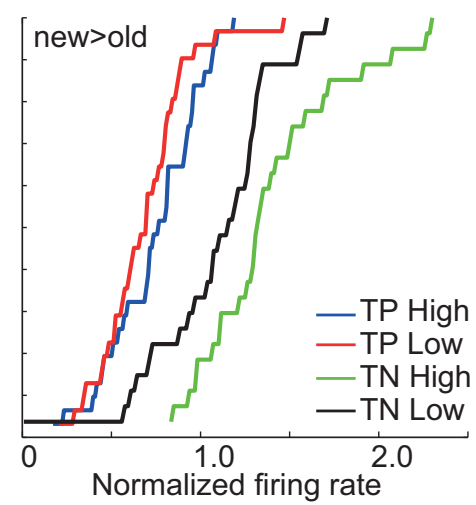

D

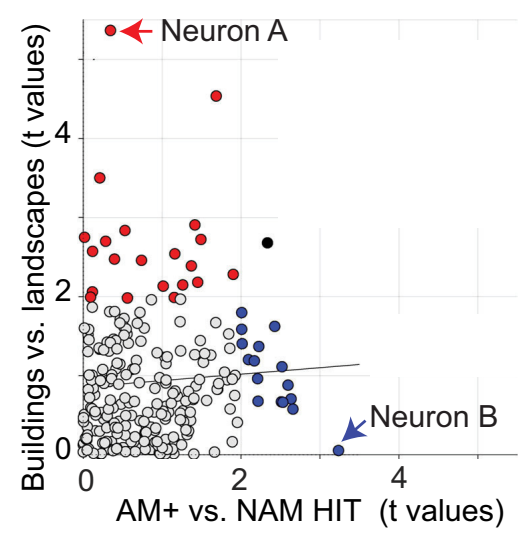

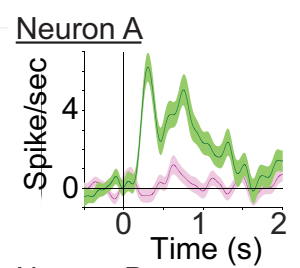

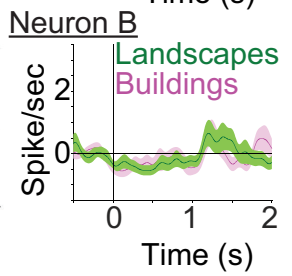

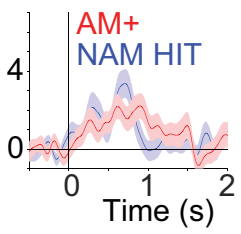

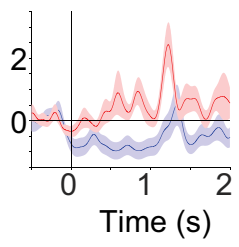

Figure 1. Encoding episodic memory strength and retrieval mode. $A$, Example memory-selective cells. Left, Cell that fires most to familiar (old) stimuli. Right, Cell that fires most to novel (new) stimuli. $\mathrm{t}=0$ is stimulus onset. Top, Raster plots. Each row represents an individual trial. Each dot represents an action potential. Bottom, Average firing rate across all trials binned 250 ms bins. $\boldsymbol{B}$, Activity of both types of memory-selective cells scale with confidence for their preferred, but not their nonpreferred, stimulus (either old [left] or new [right] stimuli). TP, True positive (old stimuli recognized correctly) shown as a cumulative density function (cdf); TN, true negative (new stimuli recognized correctly). $\boldsymbol{C}$, Orthogonal tuning to either visual category ( $x$ axis) or familiarity ( $y$ axis) during recognition memory. Each data point is a neuron. Red and green represent neurons that are primarily influenced by novelty/familiarity or category, respectively. D, Orthogonal tuning to either visual category (y axis) or successful retrieval versus familiarity only ( $x$ axis). Shown are two example neurons (marked): one only visually selective (Neuron A), the other only differentiating successful retrieval from familiarity alone (Neuron B). Each data point is a neuron. Red and blue represent neurons that are primarily influenced by visual identity and success of retrieval of the associated stimulus, respectively. A, Adapted from Faraut et al. (2018). B, C, Adapted from Rutishauser et al. (2015). D, Adapted from Staresina et al. (2019).

individual neurons in humans. Indeed, listening to a single such neuron can be highly informative about the content of memory and/or visual awareness (Rey et al., 2014; Reber et al., 2017; Staresina et al., 2019). This finding is contrary to what would be expected from a fully distributed code (Rogers and McClelland, 2014; Yuste, 2015). Instead, this work reveals that, in humans, the canonical coding principle of the single unit (Barlow, 1972) that has been used to understand sensory representation is applicable to memory.

In describing these studies, we also highlight the utility of behavioral reports. Declarative memories are memories whose content rises into awareness so that their existence can be "declared," a feature of memory that can be studied directly in humans. The importance of the phenomenological experience of retrieving an episodic memory (i.e., "mental time travel") (Boyer, 2008; Suddendorf et al., 2009) for human cognition is amply demonstrated by the profound effect of its loss because of lesions or neurodegenerative disorders. Recording from single neurons in humans offers a unique opportunity to decipher the underlying mechanisms so that, eventually, we might be able to develop new treatment modalities for these dreaded disorders of the human mind.

Building blocks of episodic memories: memory-selective cells Neurons within the human MTL signal whether stimuli are novel or familiar (Fried et al., 1997; Rutishauser et al., 2006,
2008; Viskontas et al., 2006), thereby revealing a single-neuron substrate for this core feature of episodic memory. Characterizing these neurons in detail has been a major focus for several reasons. First, a given event happens only once, thus requiring rapid plasticity. Second, successful detection of novelty is a prerequisite for encoding, thereby allowing novelty signals (Knight, 1996) to serve as signals that indicate encoding. Third, familiarity is graded: a stimulus can either be vaguely familiar with no associated attributes or be highly familiar with many associated attributes (recollection). Fourth, for a memory to be declarative, subjects need to be able to declare the presence or absence of a memory. Thus, a neuronal novelty/familiarity-related response would need to predict awareness or subjective confidence to be relevant for declarative memory. Single-neuron correlates of many of these properties have now been identified.

The response of memory-selective cells differentiates between novel and familiar stimuli. Such cells have been identified across the MTL, including the hippocampus, amygdala, entorhinal cortex, and parahippocampal cortex. Two types of memory-selective cells have been identified: a novelty type and a familiarity type, which increase their response relative to baseline for novel and familiar stimuli, respectively (Fig. 1A). The responses of these cells are compatible with the four criteria outlined above. First, they express single-trial learning (Rutishauser et al., 2006; Kamiński et al., 2018). Second, the phase-locking to theta oscillations of the novelty type of memory-selective cells is indicative of encoding 
success or failure (Rutishauser et al., 2010). Third, their response is graded, with responses accompanied by both familiarity and successful retrieval of an associated stimulus larger than those that are accompanied only by familiarity but no retrieval of a previously learned association (Fig. 1D). This effect has been demonstrated both for cued recall of paired items (Staresina et al., 2019; Derner et al., 2020) and for cued spatial source recall (Rutishauser et al., 2008). Fourth, the response of memory-selective cells differentiates between otherwise identical correct decisions that are made either with high or low subjective confidence (Fig. 1B) (Rutishauser et al., 2015). Together, this shows that the response of memory-selective cells in the human MTL are likely a reflection of episodic memory. A key next goal is to determine how the signal carried by memoryselective cells is computed and where the plasticity expressed by these cells occurs.

The response properties of memory-selective cells provide constraints on how the signal they carry might be computed. First, their response latency is long: their response differs between novel and familiar stimuli starting at $\sim 450 \mathrm{~ms}$ after stimulus onset. Relative to the response latencies of neurons in sensory neocortex, this is slow and too late for some behaviors, such as basic categorization (Thorpe et al., 1996). Instead, this long latency suggests that the signal carried by memory-selective cells is related to declarative memory, which operates at a slower timescale. Supporting this view, evoked potentials recorded with scalp EEG that are related to declarative memory (e.g., the N400 or the late negative component) occur with similarly long latencies (Mormann et al., 2005). Second, most neurons with familiarity/novelty responses in the MTL are not tuned to memory content (Fig. 1C).

In addition to the encoding of novelty/familiarity in recognition memory, memory-selective responses have also been observed during cued retrieval in associative memory tasks (Fig. $1 D)$. In this task, a type of memory-selective cell was identified that signaled whether a stimulus associated with a cue was retrieved or not, but not what the identity of the retrieved item was (Staresina et al., 2019). An overall conclusion from these recordings is that the human MTL contains an orthogonal code for memory content and familiarity (Rutishauser et al., 2015; Staresina et al., 2019; Derner et al., 2020).

\section{Building blocks of episodic memories: semantically invariant concept cells}

The response of many neurons in the human amygdala, hippocampus, entorhinal cortex, and parahippocampal cortex is related to the high-level visual interpretation of stimuli. A continuum of such cells has been discovered (for examples, see Fig. $2 A-C)$, from relatively broadly tuned, category cells to more sparsely tuned cells signaling semantic concepts, all the way to highly sparse and invariant concept cells that respond whenever a picture shows a particular individual. We refer to such cells jointly as "visually selective," but this is not meant to imply that these cells do not also respond to other modalities (Quiroga et al., 2009). The most broadly tuned type of visually selective cells is category neurons (Fig. 2C), which respond every time an image belonging to a particular broad visual category is shown (Kreiman et al., 2000). Examples of category neurons are neurons responding to faces (Minxha et al., 2017), animals (Mormann et al., 2011), outdoor scenes (Mormann et al., 2017), fruits (Minxha et al., 2020), and various other object categories (Reber et al., 2019). The response of category cells is insensitive to whether a stimulus is novel or familiar as well as to whether a stimulus was retrieved with high or low confidence (Rutishauser et al., 2015); this does not exclude short-lasting repetition suppression (Pedreira et al., 2010; Minxha et al., 2020). At the other extreme of selectivity and invariance are "concept cells," which become active briefly only when a particular high-level concept is shown (Quiroga et al., 2005). Such cells can develop relatively rapidly as demonstrated by cells that respond to experimenters previously unknown to the subject (Fig. 2B). Most concept cells respond to concepts personally known to the person the neuron was recorded from (Viskontas et al., 2009). While the response of concept cells is sparse, these cells are not rare: it has been estimated that 2-5 million MTL cells respond to a given concept (Waydo et al., 2006).

The response of visually selective cells is indicative of whether a given stimulus has entered conscious awareness of the subject as declared by subjective report (Kreiman et al., 2002; Quiroga et al., 2008, 2014; Rey et al., 2014; Reber et al., 2017). The more anterior in the MTL a concept cell is located, the more likely its activity is to covary with awareness (Reber et al., 2017; Fu and Rutishauser, 2018). The view that responses of visually selective cells are related to the current content of awareness is in addition supported by the finding that, in dense scenes, category cells signal the categorical identity of the currently attended stimulus, a finding that applies to both overt and covert attention (Minxha et al., 2017). Visually selective cells thus appear well suited to encode declarative memories, which are memories that have risen into awareness.

The time that elapses between onset of a stimulus and a differential response of visually selective cells is $\sim 300 \mathrm{~ms}$ (Mormann et al., 2008; Rutishauser et al., 2015). The latency until an individual cell starts responding is inversely proportional to its selectivity, with more selective responses occurring later (Mormann et al., 2008). This is late compared with responses in sensory areas measured using similar techniques (Self et al., 2016), but earlier than that of memory selective cells (discussed above). Also, this long latency is what is expected from a stimulus that has risen into awareness (Mashour et al., 2020) and is concordant with the known response latencies of memory-related ERPs (Mormann et al., 2005).

These data have given rise to the hypothesis that visually selective cells represent semantic memories (Rutishauser, 2019), which in turn are the building blocks of episodic memories (Quiroga, 2012). Further evidence for this proposal is provided by population-level analysis of a large set of single neurons (Reber et al., 2019). This analysis revealed that the response of visually selective cells generalized between instances of a given semantic concept. Whereas some semantic concepts represented by cells were relatively broad ("food items"), others were more narrow ("cheese," see Fig. 2A). Indeed, whether a given cell responds to a given stimulus or not could be predicted with high accuracy from the semantic label of a stimulus (Fig. $2 A$ ). Notably, the response of cells was not "all-or-nothing." Rather, the response was best described as a tuning curve in semantic space with graded responses to different instances (stimuli) from a given semantic category (e.g., a cell that responds with a different magnitude to a picture of shorts and a red jacket; see Fig. $2 \mathrm{~A}$, top row). This is a critical new insight that supports the hypothesis that visually selective cells represent semantic memories and, as such, are a core part of the engram. This view, however, is challenged by two recent studies describing responses of concept neurons to different person identities as binary rather than graded (Rey et al., 2018, 2020). Future work is needed to decipher a better understanding of the regimens under which visually selective cell responses can be conceptualized as binary versus graded, in particular whether these constitute functionally different subgroups of cells and whether these two types of cells have a differential role in declarative memory. 
A
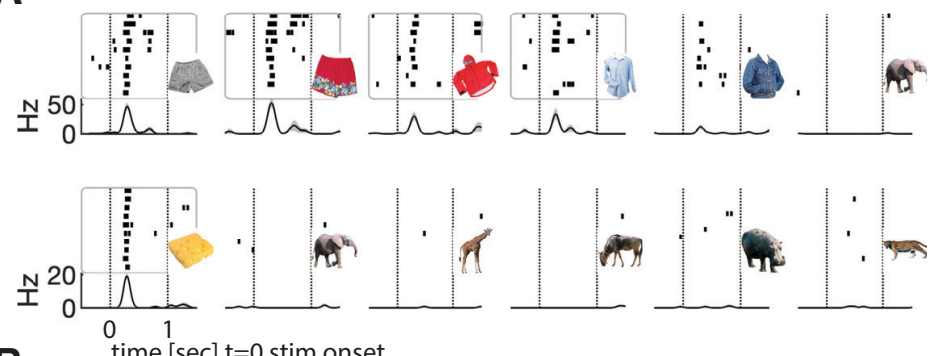

B

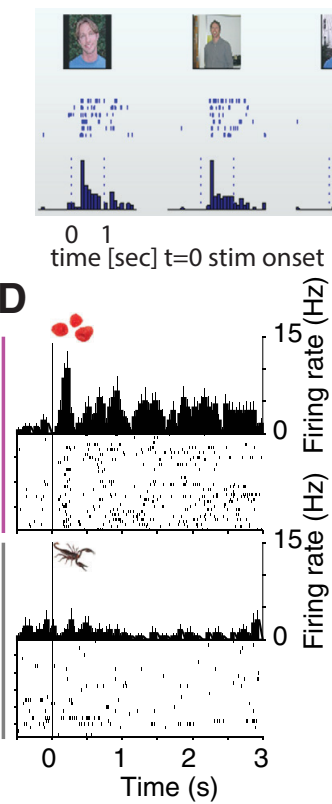

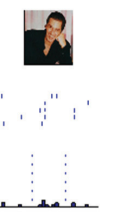
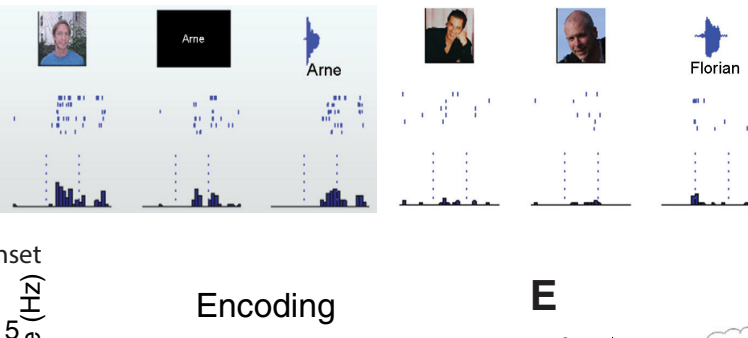

ding

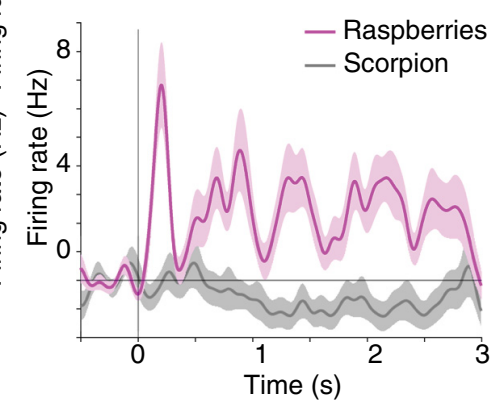

E

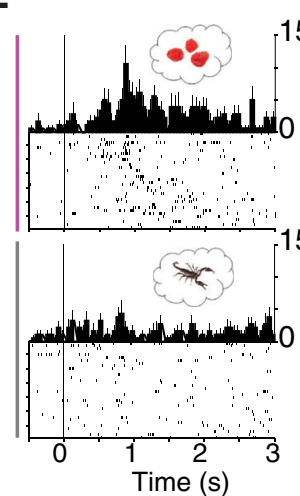

C
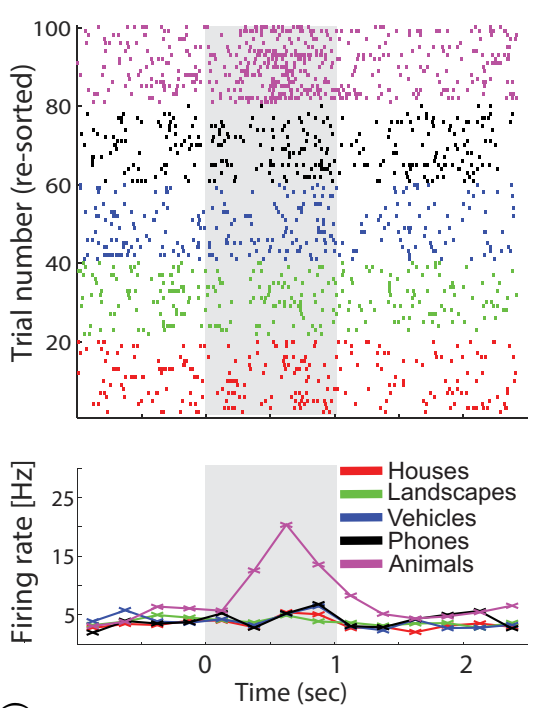

Retrieval

Figure 2. Abstract semantic representations and their role in episodic retrieval. $A-C$, Example of visually selective neurons with tuning compatible with semantic representations. $A$, Top, Visually selective neuron responding to many different images showing clothes (from hippocampus). Bottom, Visually selective neuron only responding to a single image of a food item (from amygdala). $\boldsymbol{B}$, Invariant multimodal concept neuron that responds only to images and written and spoken name of an experimenter. $\boldsymbol{C}$, Visually selective category neuron. $t=0$ is stimulus onset. Top, Raster plot represents the spiking response to different stimulus categories (indicated by color). Bottom, Average firing rate across trials in bins of $250 \mathrm{~ms}$. $\boldsymbol{D}$, Visually selective neuron in the entorhinal cortex with a response to "raspberries," but not to "scorpion," during encoding. $\boldsymbol{E}$, Same neuron as shown in $\boldsymbol{D}$, but now during cued retrieval. This neuron increased its firing selectively only if associated to-be-retrieved image was "raspberries." This is a signature of content-specific reinstatement during cued retrieval. $\boldsymbol{A}$, Adapted from Reber et al. (2019b). B, Adapted from Quiroga et al. (2009). C, Adapted from Rutishauser (2019). D, E, Adapted from Staresina et al. (2019).

\section{Encoding of associations}

Memory associates different aspects of experience with each other. For example, an episodic memory might be that I met Person X at Place Y at Time Z. Visually selective cells by themselves represent an encoding of associations: if two stimuli are conceptually related, they activate the same cell (i.e., all stimuli that belong to the same semantic concept). This property also holds for more sparsely tuned cells: when such a cell responds to several seemingly unrelated stimuli (e.g., two different basketball players that play on the same team), these stimuli are indeed conceptually related as revealed by an analysis of how often the two semantic concepts co-occur (De Falco et al., 2016). This broadening of tuning can be induced experimentally: repeated pairing of a responsive and nonresponsive concept leads to a broadening of the tuning of an individual cell, which starts to respond also to the non-response-eliciting concept as a result of learning (Ison et al., 2015). Associative encoding has also been observed in the temporal domain: if a subject learns that a picture of Concept $\mathrm{X}$ is always followed by that of Concept $\mathrm{Y}$, the cell responsive to $\mathrm{Y}$ starts firing already in the delay period before Image $\mathrm{Y}$ appears (Reddy et al., 2015). Of note, the timescale of plasticity in the data discussed above is relatively long (several trials). Thus, these changes occur on the temporal scale expected of semantic, but not episodic, memories, which rely on single-trial learning. Together, these data show that the representations formed by visually selective cells are plastic as expected from a semantic representation that supports episodic memory.

A second form of associative memory that has been studied is cued retrieval: after learning pairs (e.g., face-name pairs), one of the items (i.e., the face) is shown and the subject attempts to retrieve the paired stimulus (i.e., the name). Recordings in the entorhinal cortex (Staresina et al., 2019) reveal cells that signal the content of the retrieved stimulus (the name in the above example) (Fig. 2D,E). Reinstatement of the memory content retrieved after the cue is shown is sufficiently strong to allow decoding of the recollected memory. Indeed, earlier work has revealed that reinstatement extends beyond neurons specifically tuned to retrieved memory content: the neural state ("neural context") that was present at the time of encoding tends to be reinstated during successful recollection (Jang et al., 2017) Folkerts et al., 2018. Together, this body of work reveals a singleneuron correlate of reinstatement, thereby providing an empirical basis for a key theory about how episodic memory is organized (Howard et al., 2005; Polyn et al., 2009). 

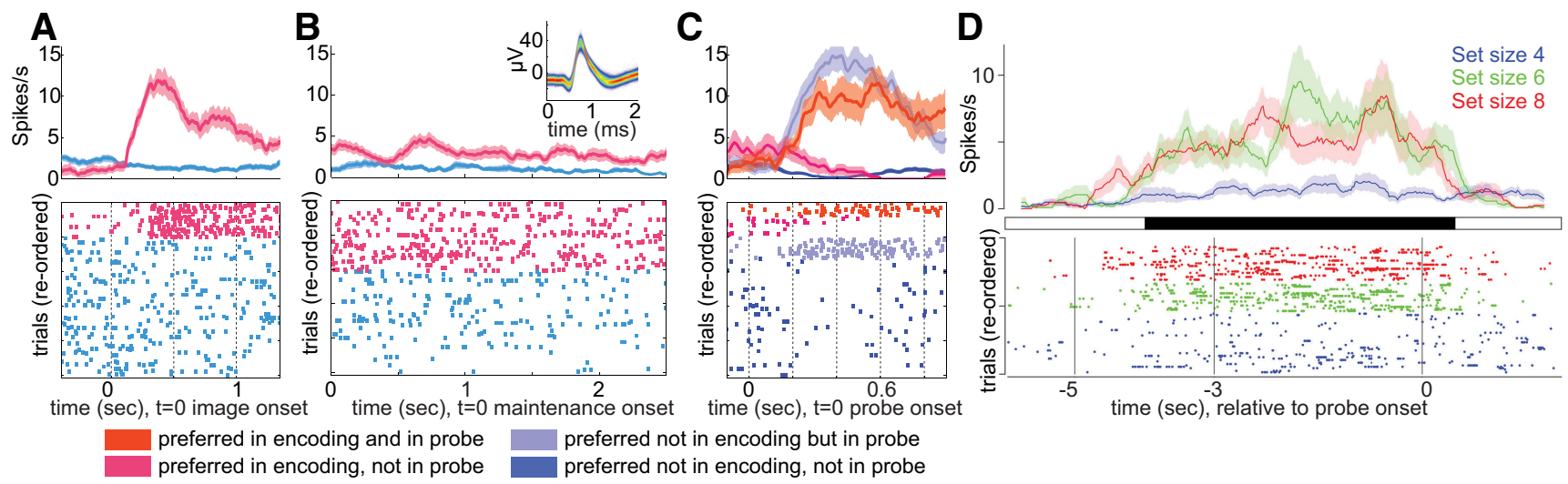

Figure 3. Persistently active cells as a substrate for WM. A-C, Example of a visually selective neuron recorded from the amygdala during a WM task with pictures as items. Shown is the encoding $(\boldsymbol{A})$, maintenance $(\boldsymbol{B})$, and probe $(\boldsymbol{C})$ period. The firing rate of this neuron increases starting during encoding when its preferred stimulus is shown (magenta) and maintains this activity during the maintenance period if its preferred image is held in memory $(\boldsymbol{B}) . \boldsymbol{B}$, Inset, The mean waveform of this neuron. $\boldsymbol{D}$, Example neuron in the hippocampus recorded during a WM task with letters as items. This neuron increases its activity during the maintenance period as a function of load but not memory content. $\boldsymbol{A}-\boldsymbol{D}$, Bottom, Raster plots represent the spiking response in individual trials (one per row), with identity of the trial marked by color. Top, Average firing rate as a function of time across all trials as a function of time. $A-C$, Adapted from Kamiński et al. (2020). D, Adapted from Boran et al. (2019).

\section{Persistent activity as a mechanism for working memory (WM)}

Some MTL cells can remain persistently active for several seconds while subjects actively hold the cell's preferred stimulus in WM, a finding first reported in 2017 independently by two groups (Kamiński et al., 2017; Kornblith et al., 2017). Decoding from such persistently active cells allows the decoding of the content of WM (Kamiński et al., 2020). Also, the cells' activity decreases as a function of memory load, as expected from a representation of short-term memory (Kamiński et al., 2017), and their response strength predicts whether the memory will later be retrieved successfully (Kornblith et al., 2017). This pattern of activation is different from that seen in a typical experiment, in which these cells show transient activation lasting $<500 \mathrm{~ms}$ after stimulus onset. During WM tasks, persistently active visually selective cells maintain their selectivity and preference, such that the stimulus to which they are selective during stimulus presentation remains the same for several seconds during maintenance, when no image is shown (Fig. $3 A-C$ ). Approximately $50 \%$ of concept cells exhibit such continuous content-selective activation. A second type of persistently active cell has been discovered in the hippocampus: cells whose persistent activity increases as a function of load but which is otherwise not content-selective (Fig. 3D). Together, these studies reveal that the substrate for long-term memories can also support WM. More broadly, this work reveals a cellular substrate for human WM: that of persistently active cells. The electrophysiological properties of these human cells appear much like those reported since the earliest such studies in the macaque frontal lobe (Fuster and Alexander, 1971) or recently in rodent MTL (Masuda et al., 2020), except that here their tuning is for high-level semantic concepts rather than spatial locations.

It might appear paradoxical that cells in the human MTL would support WM since the MTL is not strictly necessary for WM. While this is true for many tasks (Squire et al., 2004), under more challenging circumstances, such as interference from distractors or high memory load, humans with MTL lesions do exhibit WM deficits (Jeneson and Squire, 2012). Furthermore, there is evidence that the MTL may support maintenance of visuospatial relations between objects at sufficient levels of precision in WM (Alvarez and Cavanagh, 2004). Evidence for a role of the MTL in WM maintenance can also be seen at the field potential level: both sustained theta-band activity in the MTL and theta-mediated PFC-MTL interactions are prominent in the MTL during WM maintenance (Johnson et al., 2018). Based on this, we hypothesize that the persistently active MTL cells in humans support WM under these more challenging circumstances in which purely sensory representations are no longer sufficient to maintain the memory. The association of MTL visually selective cell activity with conscious awareness allows a further hypothesis: that these cells represent WM content that is presently active in awareness, an active form of WM that has long been recognized to be distinct from other forms of WM, such as activated long-term memory in theoretical models of WM (Cowan, 1988; Kamiński and Rutishauser, 2020). While many aspects of this relationship remain to be investigated, this identification of a cellular substrate of WM that is semantically selective offers many opportunities to directly test and refine these important cognitive models.

\section{From memories to decisions}

How are representations of memories in the MTL used for making decisions? Neuroimaging reveals several cortical areas that might carry memory-related choice signals (Simons and Spiers, 2003; Rugg and Vilberg, 2013), including the medial frontal cortex (MFC) and left posterior parietal cortex (Wagner et al., 2005); our focus here is on the MFC (for results on the PPC, see Rutishauser et al., 2018). In rodents, the MFC receives monosynaptic connections from hippocampal neurons, and these connections are causally relevant for making memory-based decisions during spatial navigation (Spellman et al., 2015; Tamura et al., 2017).

Neurons within the human presupplementary motor area and dorsal ACC signal decisions about whether a stimulus is novel or familiar in a recognition memory task (Minxha et al., 2020), a role distinct from the traditionally studied role of the MFC in cognitive control and performance monitoring (Fu et al., 2019). The neurons signaled these decisions in an abstract format regardless of motor output modality, indicating that their response is a reflection of an abstract choice representation rather than motor actions. The neurons that signaled memorybased decisions were largely distinct from those that signaled perceptual decisions. Also, neurons carrying memory-based choice signals flexibly adjusted their phase-locking with hippo- 
campal theta oscillations: they increased their phase-locking only when a decision required access to memory. Finally, memorybased choice cells signal the decision about the memory rather than the memory itself: in cases of errors, the activity of the cells predicted the subject's choice regardless of whether it was true or false. Together, this work reveals a mechanism by which cortical decision processes flexibly interact with the MTL.

\section{Outlook: new technology to record human single neurons?}

The recordings discussed above rely on hybrid electrodes (Cash and Hochberg, 2015) that allow recording from microwires in patients with epilepsy undergoing placement of depth electrodes for localization of their seizure focus (Babb et al., 1973). The majority of studies use variants of the Behnke-Fried electrode (Fried et al., 1999), which has been in routine use for many years. This electrode consists of 8 or 9 microwires protruding from the tip of a depth electrode. These wires exit the carrier in an uncontrolled fashion, thereby preventing their use as tetrodes to improve spike sorting or to calculate current source density. A notable development in this direction is tetrodes in humans (Despouy et al., 2020), which promise improved yield and isolation quality. A key next milestone will be to adapt and safely deploy newer highdensity recording methods that will make it possible to simultaneously record from hundreds or thousands of neurons (Juavinett et al., 2019). Taking this major step is a key challenge for the field because of the considerable effort needed to demonstrate the safety of this technology for human use.

In conclusion, in this review, we discussed two broad classes of cells in the human MTL: those that selectively respond to specific subsets of stimuli and those that differentiate between different aspects of memory. The latter group is not content-selective: they signal, for example, whether a stimulus is novel or familiar, or whether an associated attribute was retrieved or not. The activity of these cells changes after a single exposure and predicts aspects of declared subjective human experience, such as confidence and the experience of recollection. In models of declarative memory (Yonelinas, 2001; Wixted, 2007), decision variables that signal "memory strength" that are otherwise content-free are a common assumption. The signals carried by memory-selective cells in the MTL share many of the properties predicted by such models, thereby enabling a close interaction between theory and circuit-level experiments.

Are visually selective cells in the human MTL a kind of a "place cell" (as found in rodents) in the cognitive space formed by our semantic memories (Lisman et al., 2017)? While there are many striking similarities (for details, see Quiroga, 2012), there are also salient differences that call this analogy into question. A remarkable feature of visually selective cells in humans is their context independence, including being active during the very first exposure to a novel stimulus. This is unlike place cells in rodents and nonhuman primates, which are highly dependent on context and behavior (Anderson and Jeffery, 2003; Courellis et al., 2019). For example, place cell firing depends on direction of movement, and modifications of the environment or task goals can lead to complete remapping. While this question needs more systematic study, there are critical differences between the properties of these two types of cells.

Whole-brain fMRI studies have revealed remarkable patterns of semantic tuning that tile the human cortex (Huth et al., 2016). These patterns are seen at different levels of semantic abstraction, which is reminiscent of how visually selective cells respond to semantic concepts. An intriguing open question is whether these two findings, made at very different scales of observation, are an expression of the same phenomena. Future work is needed to determine whether the trials on which a semantically tuned cell in the MTL responds are the same trials as those on which the corresponding parts of cortex become active as assessed by fMRI. If so, this might indicate that VS cells in the MTL that are semantically tuned are cells that "index" or "point" to cortical patterns of activity that represent memory content, a mode of hippocampal operation long theorized (Teyler and Rudy, 2007).

Last, it is imperative to point out that, without work performed in animal models, the rare and select opportunities to record invasively from humans (which gave rise to all the results discussed here) could not be used meaningfully. Indeed, these two approaches are complementary: a key role for human invasive experiments is to establish a bridge between species by establishing the relevance of certain phenomena for human memory, behavior, and disease. As a result of such links, the identified mechanisms can then be invested in detail at the molecular and cellular levels in model animals (Kandel et al., 2014) in ways that are impossible or unethical in humans but, at the same time, are of direct relevance because of the established links between the species.

\section{References}

Alvarez GA, Cavanagh P (2004) The capacity of visual short-term memory is set both by visual information load and by number of objects. Psychol Sci 15:106-111.

Anderson MI, Jeffery KJ (2003) Heterogeneous modulation of place cell firing by changes in context. J Neurosci 23:8827-8835.

Babb TL, Carr E, Crandall PH (1973) Analysis of extracellular firing patterns of deep temporal lobe structures in man. Electroencephalogr Clin Neurophysiol 34:247-257.

Barlow HB (1972) Single units and sensation: a neuron doctrine for perceptual psychology? Perception 1:371-394.

Boran E, Fedele T, Klaver P, Hilfiker P, Stieglitz L, Grunwald T, Sarnthein J (2019) Persistent hippocampal neural firing and hippocampal-cortical coupling predict verbal working memory load. Sci Adv 5:eaav3687.

Boyer P (2008) Evolutionary economics of mental time travel? Trends Cogn Sci 12:219-224.

Cash SS, Hochberg LR (2015) The emergence of single neurons in clinical neurology. Neuron 86:79-91.

Courellis HS, Nummela SU, Metke M, Diehl GW, Bussell R, Cauwenberghs G, Miller CT (2019) Spatial encoding in primate hippocampus during free navigation. PLoS Biol 17:e3000546.

Cowan N (1988) Evolving conceptions of memory storage, selective attention, and their mutual constraints within the human information-processing system. Psychol Bull 104:163-191.

De Falco E, Ison MJ, Fried I, Quiroga R (2016) Long-term coding of personal and universal associations underlying the memory web in the human brain. Nat Commun 7:13408.

Derner M, Dehnen G, Chaieb L, Reber TP, Borger V, Surges R, Staresina BP, Mormann F, Fell J (2020) Patterns of single-neuron activity during associative recognition memory in the human medial temporal lobe. Neuroimage 221:117214.

Despouy E, Curot J, Reddy L, Nowak LG, Deudon M, Sol JC, Lotterie JA, Denuelle M, Maziz A, Bergaud C, Thorpe SJ, Valton L, Barbeau EJ (2020) Recording local field potential and neuronal activity with tetrodes in epileptic patients. J Neurosci Methods 341:108759.

Eichenbaum H, Yonelinas AP, Ranganath C (2007) The medial temporal lobe and recognition memory. Annu Rev Neurosci 30:123-152.

Engel AK, Moll CK, Fried I, Ojemann GA (2005) Invasive recordings from the human brain: clinical insights and beyond. Nat Rev Neurosci 6:3547.

Faraut MC, Carlson AA, Sullivan S, Tudusciuc O, Ross I, Reed CM, Chung JM, Mamelak AN, Rutishauser U (2018) Dataset of human medial temporal lobe single neuron activity during declarative memory encoding and recognition. Sci Data 5:180010. 
Folkerts S, Rutishauser U, Howard MW (2018) Human episodic memory retrieval is accompanied by a neural contiguity effect. J. Neurosci, 38:42004211.

Fried I, MacDonald KA, Wilson CL (1997) Single neuron activity in human hippocampus and amygdala during recognition of faces and objects. Neuron 18:753-765.

Fried I, Rutishauser U, Cerf M, Kreiman G (2014) Single neuron studies of the human brain: probing cognition. Boston: Massachusetts Institute of Technology.

Fried I, Wilson CL, Maidment NT, Engel J Jr, Behnke E, Fields TA, MacDonald KA, Morrow JW, Ackerson L (1999) Cerebral microdialysis combined with single-neuron and electroencephalographic recording in neurosurgical patients: technical note. J Neurosurg 91:697-705.

Fu Z, Rutishauser U (2018) Single-neuron correlates of awareness during attentional blinks. Trends Cogn Sci 22:5-7.

Fu Z, Wu DJ, Ross I, Chung JM, Mamelak AN, Adolphs R, Rutishauser U (2019) Single-neuron correlates of error monitoring and post-error adjustments in human medial frontal cortex. Neuron 101:165-177.e165.

Fuster JM, Alexander GE (1971) Neuron activity related to short-term memory. Science 173:652-654.

Howard MW, Fotedar MS, Datey AV, Hasselmo ME (2005) The temporal context model in spatial navigation and relational learning: toward a common explanation of medial temporal lobe function across domains. Psychol Rev 112:75-116.

Huth AG, de Heer WA, Griffiths TL, Theunissen FE, Gallant JL (2016) Natural speech reveals the semantic maps that tile human cerebral cortex. Nature 532:453-458.

Ison MJ, Quiroga R, Fried I (2015) Rapid encoding of new memories by individual neurons in the human brain. Neuron 87:220-230.

Jang AI, Wittig JH Jr, Inati SK, Zaghloul KA (2017) Human cortical neurons in the anterior temporal lobe reinstate spiking activity during verbal memory retrieval. Curr Biol 27:1700-1705. e1705.

Jeneson A, Squire LR (2012) Working memory, long-term memory, and medial temporal lobe function. Learn Mem 19:15-25.

Johnson EL, Adams JN, Solbakk AK, Endestad T, Larsson PG, Ivanovic J, Meling TR, Lin JJ, Knight RT (2018) Dynamic frontotemporal systems process space and time in working memory. PLoS Biol 16:e2004274.

Johnson EL, Knight RT (2015) Intracranial recordings and human memory. Curr Opin Neurobiol 31:18-25.

Juavinett AL, Bekheet G, Churchland AK (2019) Chronically implanted Neuropixels probes enable high-yield recordings in freely moving mice. Elife 8:e47188.

Kamiński J, Rutishauser U (2020) Between persistently active and activitysilent frameworks: novel vistas on the cellular basis of working memory. Ann NY Acad Sci 1464:64-75.

Kamiński J, Sullivan S, Chung JM, Ross IB, Mamelak AN, Rutishauser U (2017) Persistently active neurons in human medial frontal and medial temporal lobe support working memory. Nat Neurosci 20:590-601.

Kamiński J, Mamelak AN, Birch K, Mosher CP, Tagliati M, Rutishauser U (2018) Novelty-sensitive dopaminergic neurons in the human substantia nigra predict success of declarative memory formation. Curr Biol 28:1333-1343.e1334.

Kamiński J, Brzezicka A, Mamelak AN, Rutishauser U (2020) Combined phase-rate coding by persistently active neurons as a mechanism for maintaining multiple items in working memory in humans. Neuron 106:256-264.e253.

Kandel ER, Dudai Y, Mayford MR (2014) The molecular and systems biology of memory. Cell 157:163-186.

Knight R (1996) Contribution of human hippocampal region to novelty detection. Nature 383:256-259.

Kornblith S, Quiroga R, Koch C, Fried I, Mormann F (2017) Persistent single-neuron activity during working memory in the human medial temporal lobe. Curr Biol 27:1026-1032.

Kreiman G, Koch C, Fried I (2000) Category-specific visual responses of single neurons in the human medial temporal lobe. Nat Neurosci 3:946953.

Kreiman G, Fried I, Koch C (2002) Single-neuron correlates of subjective vision in the human medial temporal lobe. Proc Natl Acad Sci USA 99:8378-8383.

Lisman J, Buzsaki G, Eichenbaum H, Nadel L, Ranganath C, Redish AD (2017) Viewpoints: how the hippocampus contributes to memory, navigation and cognition. Nat Neurosci 20:1434-1447.
Manns JR, Hopkins RO, Squire LR (2003) Semantic memory and the human hippocampus. Neuron 38:127-133.

Mashour GA, Roelfsema P, Changeux JP, Dehaene S (2020) Conscious processing and the global neuronal workspace hypothesis. Neuron 105:776798.

Masuda A, Sano C, Zhang Q, Goto H, McHugh TJ, Fujisawa S, Itohara S (2020) The hippocampus encodes delay and value information during delay-discounting decision making. eLife 9:e52466.

Minxha J, Mosher C, Morrow JK, Mamelak AN, Adolphs R, Gothard KM, Rutishauser U (2017) Fixations gate species-specific responses to free viewing of faces in the human and macaque amygdala. Cell Rep 18:878891.

Minxha J, Adolphs R, Fusi S, Mamelak AN, Rutishauser U (2020) Flexible recruitment of memory-based choice representations by the human medial frontal cortex. Science 368:eaba3313.

Mormann F, Fell J, Axmacher N, Weber B, Lehnertz K, Elger CE, Fernandez G (2005) Phase/amplitude reset and theta-gamma interaction in the human medial temporal lobe during a continuous word recognition memory task. Hippocampus 15:890-900.

Mormann F, Kornblith S, Quiroga RQ, Kraskov A, Cerf M, Fried I, Koch C (2008) Latency and selectivity of single neurons indicate hierarchical processing in the human medial temporal lobe. J Neurosci 28:8865-8872.

Mormann F, Dubois J, Kornblith S, Milosavljevic M, Cerf M, Ison M, Tsuchiya N, Kraskov A, Quiroga RQ, Adolphs R, Fried I, Koch C (2011) A category-specific response to animals in the right human amygdala. Nat Neurosci 14:1247-1249.

Mormann F, Kornblith S, Cerf M, Ison MJ, Kraskov A, Tran M, Knieling S, Quiroga R, Koch C, Fried I (2017) Scene-selective coding by single neurons in the human parahippocampal cortex. Proc Natl Acad Sci USA 114:1153-1158.

Pedreira C, Mormann F, Kraskov A, Cerf M, Fried I, Koch C, Quiroga RQ (2010) Responses of human medial temporal lobe neurons are modulated by stimulus repetition. J Neurophysiol 103:97-107.

Polyn SM, Norman KA, Kahana MJ (2009) A context maintenance and retrieval model of organizational processes in free recall. Psychol Rev 116:129-156.

Quiroga RQ (2012) Concept cells: the building blocks of declarative memory functions. Nat Rev Neurosci 13:587-597.

Quiroga R (2019) Plugging in to human memory: advantages, challenges, and insights from human single-neuron recordings. Cell 179:1015-1032.

Quiroga RQ, Reddy L, Kreiman G, Koch C, Fried I (2005) Invariant visual representation by single neurons in the human brain. Nature 435:11021107.

Quiroga RQ, Mukamel R, Isham EA, Malach R, Fried I (2008) Human single-neuron responses at the threshold of conscious recognition. Proc Natl Acad Sci USA 105:3599-3604.

Quiroga R, Kraskov A, Koch C, Fried I (2009) Explicit encoding of multimodal percepts by single neurons in the human brain. Curr Biol 19:13081313.

Quiroga RQ, Kraskov A, Mormann F, Fried I, Koch C (2014) Single-cell responses to face adaptation in the human medial temporal lobe. Neuron 84:363-369.

Reber TP, Faber J, Niediek J, Bostrom J, Elger CE, Mormann F (2017) Singleneuron correlates of conscious perception in the human medial temporal lobe. Curr Biol 27:2991-2998.e2992.

Reber TP, Bausch M, Mackay S, Bostrom J, Elger CE, Mormann F (2019) Representation of abstract semantic knowledge in populations of human single neurons in the medial temporal lobe. PLoS Biol 17:e3000290.

Reddy L, Poncet M, Self MW, Peters JC, Douw L, van Dellen E, Claus S, Reijneveld JC, Baayen JC, Roelfsema PR (2015) Learning of anticipatory responses in single neurons of the human medial temporal lobe. Nat Commun 6:8556

Rey HG, Fried I, Quiroga R (2014) Timing of single-neuron and local field potential responses in the human medial temporal lobe. Curr Biol 24:299-304.

Rey HG, De Falco E, Ison MJ, Valentin A, Alarcon G, Selway R, Richardson MP, Quiroga R (2018) Encoding of long-term associations through neural unitization in the human medial temporal lobe. Nat Commun 9:4372.

Rey HG, Gori B, Chaure FJ, Collavini S, Blenkmann AO, Seoane P, Seoane E, Kochen S, Quiroga R (2020) Single neuron coding of identity in the human hippocampal formation. Curr Biol 30:1152-1159.e1153. 
Rogers TT, McClelland JL (2014) Parallel distributed processing at 25: further explorations in the microstructure of cognition. Cogn Sci 38:1024-1077.

Rugg MD, Vilberg KL (2013) Brain networks underlying episodic memory retrieval. Curr Opin Neurobiol 23:255-260.

Rutishauser U (2019) Testing models of human declarative memory at the single-neuron level. Trends Cogn Sci 23:510-524.

Rutishauser U, Mamelak AN, Schuman EM (2006) Single-trial learning of novel stimuli by individual neurons of the human hippocampus-amygdala complex. Neuron 49:805-813.

Rutishauser U, Schuman EM, Mamelak AN (2008) Activity of human hippocampal and amygdala neurons during retrieval of declarative memories. Proc Natl Acad Sci USA 105:329-334.

Rutishauser U, Ross IB, Mamelak AN, Schuman EM (2010) Human memory strength is predicted by theta-frequency phase-locking of single neurons. Nature 464:903-907.

Rutishauser U, Ye S, Koroma M, Tudusciuc O, Ross IB, Chung JM, Mamelak AN (2015) Representation of retrieval confidence by single neurons in the human medial temporal lobe. Nat Neurosci 18:1041-1050.

Rutishauser U, Aflalo T, Rosario ER, Pouratian N, Andersen RA (2018) Single-neuron representation of memory strength and recognition confidence in left human posterior parietal cortex. Neuron 97:209-220.e203.

Self MW, Peters JC, Possel JK, Reithler J, Goebel R, Ris P, Jeurissen D, Reddy L, Claus S, Baayen JC, Roelfsema PR (2016) The effects of context and attention on spiking activity in human early visual cortex. PLoS Biol 14: e1002420.

Simons JS, Spiers HJ (2003) Prefrontal and medial temporal lobe interactions in long-term memory. Nat Rev Neurosci 4:637-648.

Spellman T, Rigotti M, Ahmari SE, Fusi S, Gogos JA, Gordon JA (2015) Hippocampal-prefrontal input supports spatial encoding in working memory. Nature 522:309-314.

Squire LR, Stark CE, Clark RE (2004) The medial temporal lobe. Annu Rev Neurosci 27:279-306.
Staresina BP, Reber TP, Niediek J, Bostrom J, Elger CE, Mormann F (2019) Recollection in the human hippocampal-entorhinal cell circuitry. Nat Commun 10:1503.

Suddendorf T, Addis DR, Corballis MC (2009) Mental time travel and the shaping of the human mind. Philos Trans R Soc Lond B Biol Sci 364: 1317-1324.

Suthana N, Fried I (2012) Percepts to recollections: insights from single neuron recordings in the human brain. Trends Cogn Sci 16:427-436.

Tamura M, Spellman TJ, Rosen AM, Gogos JA, Gordon JA (2017) Hippocampal-prefrontal theta-gamma coupling during performance of a spatial working memory task. Nat Commun 8:2182.

Teyler TJ, Rudy JW (2007) The hippocampal indexing theory and episodic memory: updating the index. Hippocampus 17:1158-1169.

Thorpe S, Fize D, Marlot C (1996) Speed of processing in the human visual system. Nature 381:520-522.

Tulving E (2002) Episodic memory: from mind to brain. Annu Rev Psychol 53:1-25.

Viskontas IV, Knowlton BJ, Steinmetz PN, Fried I (2006) Differences in mnemonic processing by neurons in the human hippocampus and parahippocampal regions. J Cogn Neurosci 18:1654-1662.

Viskontas IV, Quiroga RQ, Fried I (2009) Human medial temporal lobe neurons respond preferentially to personally relevant images. Proc Natl Acad Sci USA 106:21329-21334.

Wagner AD, Shannon BJ, Kahn I, Buckner RL (2005) Parietal lobe contributions to episodic memory retrieval. Trends Cogn Sci 9:445-453.

Waydo S, Kraskov A, Quiroga R, Fried I, Koch C (2006) Sparse representation in the human medial temporal lobe. J Neurosci 26:10232-10234.

Wixted JT (2007) Dual-process theory and signal-detection theory of recognition memory. Psychol Rev 114:152-176.

Yonelinas AP (2001) Components of episodic memory: the contribution of recollection and familiarity. Philos Trans R Soc Lond B Biol Sci 356: 1363-1374.

Yuste R (2015) From the neuron doctrine to neural networks. Nat Rev Neurosci 16:487-497. 